\title{
Factores de crecimiento en la región económica número VII de Hidalgo: un análisis sectorial
}

\author{
Growth factors in the economic region number VII of Hidalgo a sector analysis
}

\author{
Gustavo Castelán González ${ }^{a}$, Jozelín María Soto Alarcón ${ }^{b}$, Eduardo Rodríguez Juárez $^{c}$
}

\begin{abstract}
:
The neoliberalism as a hegemonic current worldwide has promoted through its ideas the free market as a dogma of good economy. In Mexico, after the 80's debt crisis, there is a change in government policy and the welfare state is abandoned by the market economy. More than 30 years after the implementation of neoliberal ideas, the country is experiencing a situation of economic instability, high unemployment, job insecurity, high concentration of wealth and poverty. The benefits derived from the model have been concentrated in few hands and in few economic sectors, mainly in those that promote the generation of export products. Support to exporting companies has impaired the generation of goods focused on the domestic market, abandonment to the field in favor of the export manufacturing industry has increased the vulnerability of Mexican workers. Therefore, the objective of this work was to show that, in the context of neoliberalism in region VII of the Hidalgo State, dynamic export sectors have been favored over local sectors, which has caused a deterioration in local production. For this, the Shift and Share technique was applied to collect the degree of specialization of the region in the years 2009 and 2014. The results indicate that the region number VII of the state of Hidalgo has lost competitiveness and has not managed to grow in the same way in which the entity has done it. In addition, only the manufacturing sector has grown in all the municipalities that make up the region, impacted by the dynamics of the sector itself, which is consistent with the country's export dynamics.
\end{abstract}

Keywords:

Neoliberalism, regional economic growth, Shift-Share

Resumen:

El neoliberalismo como corriente hegemónica a nivel mundial, ha impulsado a través de sus ideas el libre mercado como dogma de buena economía. En México, después de la crisis de la deuda en la década de los 80’s, se presenta un cambio en la política de gobierno, y se abandona el Estado de bienestar por la economía de mercado. A más de 30 años de la implementación de las ideas neoliberales, el país vive una situación de inestabilidad económica, alto desempleo, precariedad laboral, alta concentración de la riqueza y pobreza. Los beneficios derivados del modelo se han concentrado en pocas manos y en pocos sectores económicos, principalmente en aquellos que promueven la generación de productos de exportación. El apoyo a las empresas exportadoras ha perjudicado la generación de bienes enfocados al mercado interno, el abandono al campo en pro de la industria maquiladora de exportación ha incrementado la vulnerabilidad de los trabajadores mexicanos. Por lo anterior, el objetivo de este trabajo fue mostrar, que en el contexto del neoliberalismo en la región VII del estado de Hidalgo, se han favorecido sectores dinámicos exportadores sobre los sectores locales, lo que ha ocasionado un deterioro en la producción regional. Se aplicó la técnica "Shift and Share" para recoger el grado de especialización de la región en los años 2009 y 2014. Los resultados indican que la región número VII del estado de Hidalgo ha perdido competitividad y no ha logrado crecer de la misma forma en que lo ha hecho la entidad. Además, sólo el sector manufacturero (maquilador) ha tenido un crecimiento en todos los municipios que conforman la región, impactado por la propia dinámica del sector, lo que es congruente con la dinámica exportadora del país.

\section{Palabras Clave:}

Neoliberalismo, Economía regional, Shift-Share

\footnotetext{
a Estudiante de la Licenciatura en Economía, Universidad Autónoma del Estado de Hidalgo, Instituto de Ciencias Económico Administrativas, Área Académica de Economía, https://orcid.org/ 0000-0002-1712-9834. Email: castelan2015@ hotmail.com

b Profesora Investigadora, Universidad Autónoma del Estado de Hidalgo, Instituto de Ciencias Económico Administrativas, área Académica de Economía, https://orcid.org/ 0000-0003-3931-9834,Email: jmsoto@uaeh.edu.mx

Autor de Correspondencia, Universidad Autónoma del Estado de Hidalgo, Instituto de Ciencias Económico Administrativas, área
} Académica de Economía, https://orcid.org/ 0000-0002-2232-7544,Email: eduardor@uaeh.edu.mx 


\section{Introducción}

El neoliberalismo es resultado de un proceso históricoevolutivo en materia económica, política y social. Retoma diversas teorías de diferentes corrientes del pensamiento, filosófico, económico y político, para construir sus hipótesis y describir el comportamiento de los seres humanos. En el ámbito económico el pensamiento neoliberal estipula que es el mercado quien asegura que a cada factor de producción se le retribuya acorde a su productividad marginal, de tal forma que se pone en juicio la necesidad de las instituciones de seguridad social y de los sindicatos en la determinación de los precios y salarios [1]. Según Méndez (1998), este término se le atribuye a Hayek y Friedman y entre las principales ideas que defienden estos autores, se encuentra que el Estado no debe intervenir en la economía, sólo debe garantizar la libre competencia de los mercados y estimularla, dar prioridad al mercado internacional sobre el interno [2].

De acuerdo con Salas-Luévano (2009), fue en la década de los 80's cuando comenzó la implementación del modelo neoliberal en México, ocasionando un abandono de la política de protección social dirigida por el Estado a una política autorregulada por el mercado [3]. El cambio de paradigma en el modelo económico trajo consigo una transformación, en la forma de entender los problemas locales, la política impulsora de polos de desarrollo dirigida por el Estado se abandona, y se sustituye por una política macroeconómica global abandonando las características regionales y potencialidades de cada localidad, municipio o entidad federativa.

La implementación del neoliberalismo en México, originó que en la sociedad mexicana se acentuaran los problemas de pobreza, desigualdad del ingreso, desempleo y vulnerabilidad social. Además, de ocasionar una polarización económica perfectamente identificada entre las regiones que conforman el país (norte y sur). Las diferencias regionales denotan cada vez más la forma en la cual se han beneficiado sectores exportadores considerados estratégicos para el desarrollo del país, olvidando aquellos que son intensivos en mano de obra y baja especialización tecnológica (como el campo, por ejemplo).

Al respecto, el estado de Hidalgo, es una entidad que no es ajena a las características que se presentan en el contexto nacional. Sus altos índices de pobreza, lo ubican como la doceava entidad más pobre del país [4], con altas disparidades regionales, principalmente entre los municipios del sur y del norte de la entidad, motivo por el cual este trabajo tuvo como objetivo mostrar, que en el contexto del neoliberalismo en la región VII del estado de
Hidalgo, se han favorecido sectores dinámico exportadores sobre los sectores locales, lo que ha ocasionado un deterioro de la producción local. Para ello, Se aplicó la técnica Shift and Share [5] para recoger el grado de especialización de la región en los años 2009 y 2014. Con el fin de poder generar un insumo para la construcción de política económica a nivel regional, y con ello puedan mitigarse las diferencias regionales de estos municipios hidalguenses.

La creación de insumos que reflejen las problemáticas regionales favorece el desarrollo regional. MerchandRojas (2007), señala que las políticas regionales tienen tres objetivos básicos: 1. desarrollar las áreas atrasadas; 2. reducir los desequilibrios y; 3 . apoyar las regiones con problemas sectoriales [6]. La región objeto de estudio de este trabajo se conforma por siete municipios, a saber, Actopan, El Arenal, Francisco I. Madero, Mixquiahuala de Juárez, Progreso de Obregón, San Salvador y Santiago de Anaya. Esta región es la numero VII de un grupo de diecisiete regiones económicas creadas por decreto para el estado de Hidalgo en el año 2011 durante la gestión del exgobernador José Francisco Olvera Ruiz [7].

El trabajo se estructura de la siguiente manera, como primer punto se presenta un análisis sobre el neoliberalismo como doctrina del pensamiento económico, identificando a sus principales exponentes y aportes al pensamiento, para así, poder explicar el surgimiento del neoliberalismo en México tema de nuestro segundo apartado. La parte tres, expone la importancia de la economía regional y las diferencias fundamentales con la idea neoliberal de no intervención, con ello se busca justificar la planeación regional como elemento de desarrollo. En el cuarto apartado se presenta la evidencia estadística y el uso de la técnica Shift and Share, para analizar los sectores de crecimiento de la región VII del estado de Hidalgo. Por último, se presentan algunas consideraciones finales $y$, las fuentes de información utilizadas para el desarrollo del presente trabajo.

\section{El Neoliberalismo}

Se ha definido al neoliberalismo como "un proyecto de clase camuflado bajo una proteica retórica sobre la libertad individual, el albedrío, la responsabilidad personal, la privatización y el libre mercado" [8]. Este término se sustenta en las ideas plasmadas por Adam Smith en 1776, en su obra Investigación sobre la naturaleza y causa de la riqueza de las naciones en la cual manifiesta su gusto y defensa por el liberalismo económico. Smith, consideraba negativo el intervencionismo estatal en la economía y promovía la ideología del libre mercado del sistema capitalista. 
Por otro lado, la escuela neoclásica es una corriente de la economía que se desarrolló entre los años 1870 y 1914 [9]. Ésta escuela asume que son los mercados los mecanismos a través de los cuales se rige el funcionamiento óptimo de la economía y que los bienes adquieren su valor en función de la utilidad marginal que generan a los consumidores, de esta manera, el liberalismo junto con el neoclasismo representa la base del pensamiento neoliberal.

El neoliberalismo defiende algunos conceptos filosóficos del liberalismo clásico del siglo XIX, sin embargo, la diferencia entre ambos radica en los alineamientos políticos y el apego hacia ideas posteriores que el propio neoliberalismo ha adoptado. De acuerdo a Harvey (2007, pág. 8):

"El neoliberalismo es, ante todo, una teoría de prácticas político-económicas que afirma que la mejor manera de promover el bienestar del ser humano, consiste en no restringir el libre desarrollo de las capacidades y de las libertades empresariales del individuo dentro de un marco institucional caracterizado por derechos de propiedad privada, fuertes mercados libres y libertad de comercio" [8].

Dentro de las principales alternativas para generar una economía sólida promovidas por el neoliberalismo se encuentran la extensión de la iniciativa privada a todas las áreas de la actividad económica o la limitación del papel del Estado, el cual después del auge del keynesianismo a finales de la década de los 70's había demostrado su incapacidad para fomentar el crecimiento y la estabilidad económica, lo que represento un parteaguas que abriría el camino a los neoliberales [1].

De esta manera la visión mundial de la economía comenzó a centrar su atención en nuevas doctrinas que fortalecían las ideas liberales, como el monetarismo de Millton Friedman [10], premio nobel de economía 1976, con aportes notables principalmente sobre teoría monetaria. Así mismo, la escuela austriaca representada por Frederick Von Hayek, considerado el principal defensor del neoliberalismo, acentúan el daño de la intervención del Estado en la economía y en su obra Camino de servidumbre, se muestra como un defensor de la libertad económica al mencionar que:

"Estar sometidos a control en nuestra actividad económica significa estar siempre controlados si no declaramos nuestro objetivo particular. Pero como, al declararlo, éste tiene que someterse también a aprobación, en realidad estamos intervenidos en todo." [11].
Las principales características del neoliberalismo se atribuyen a Hayek y Friedman [2], mismas que se mencionan a continuación:

$\checkmark$ El estado no debe intervenir en la economía sólo debe garantizar la libre competencia de los mercados y estimularla.

$\checkmark \quad$ El libre flujo de capitales, personas entre países, la circulación de mercancías, incentivando la apertura comercial.

$\checkmark$ Se prioriza al mercado internacional sobre el mercado interno.

$\checkmark \quad$ Los principales objetivos de la política económica son generar crecimiento económico, equilibrio comercial, gubernamental y financiero.

En conclusión, el neoliberalismo es resultado de un proceso histórico evolutivo en materia económica, política y social, que retoma diversas teorías que anteponen el individualismo al colectivismo. Para los teóricos neoliberales es el mercado quien asegura que a cada factor de producción se le retribuya acorde a su productividad, de tal forma que se pone en juicio la necesidad de las instituciones de seguridad social, de los sindicatos y de cualquier elemento ajeno al mercado para garantizar el bienestar social [1], convirtiéndose en dogma de buena economía.

\section{EI Neoliberalismo en México}

Desde los años cuarenta, el proteccionismo hacia el mercado interno a través de la aplicación de barreras arancelarias y no arancelarias fue la acción estratégica aplicada por el Estado para impulsar el desarrollo en México. La caída del modelo de crecimiento económico basado en la Industrialización por Sustitución de Importaciones (ISI), justificada a partir de la crisis de deuda de la economía mexicana, dio paso al comienzo del modelo neoliberal en el país.

Las bases del neoliberalismo en México comenzaron a establecerse durante el sexenio de Miguel de la Madrid (1982-1988), con la venta de las primeras empresas paraestatales [3]. En el trabajo de Méndez (1998), señala que durante la década de los años ochenta el gobierno mexicano deseaba la inserción en la economía mundial, esto se logró a través de la incorporación de México al GATT (Acuerdo General sobre Aranceles Aduaneros y Comercio). Fue en el año 1994 que el proceso de apertura y liberalización económica se concretó tras la entrada en vigor del Tratado de Libre Comercio de América del Norte, (TLCAN) en la cual participaron en conjunto Estados Unidos, Canadá y México, sin embargo, el TLCAN promovía el flujo de mercancías y capitales entre los tres países, pero no así el flujo de personas [2].

El proceso de desmantelamiento de la infraestructura nacional abarca los periodos de mandato de los ex 
presidentes Carlos Salinas de Gortari (1988-1994), establecimiento de las bases para la privatización y firma del TLCAN; Ernesto Zedillo (1994-2000), venta de empresas públicas y entrada en vigor del TLCAN, Vicente Fox Quezada (2000-2006) fomentó la intervención del capital extranjero, con acciones como intentos por privatizar la industria energética, a lo que daría seguimiento, Felipe Calderón Hinojosa (2006-2012) [3]. Enrique Peña Nieto (2012-2018), implementación de reformas estructurales como la reforma energética y laboral.

La implementación del modelo neoliberal en México, ha fomentado una política de crecimiento basada en las exportaciones, y que tiene como eje de competitividad el salario. Los bajos salarios han ocasionado el detrimento del poder adquisitivo de los trabajadores mexicanos, además los procesos de privatización de las empresas públicas y la desaparición del Estado de bienestar ha provocado un incremento considerable en el número de trabajadores desempleados 0 en la informalidad, aumentando el número de mexicanos que se encuentran en pobreza y haciéndolos cada vez más vulnerables.

Es claro que el neoliberalismo en México no puede vislumbrarse como un modelo de desarrollo exitoso, pues existe una excesiva concentración de la riqueza en pocas manos, además, de un gusto por parte de la política de gobierno de favorecer sectores dinámicos exportadores, deteriorando el mercado interno y provocando, desempleo, trabajo precario, marginación y pobreza en un amplio sector de la sociedad mexicana.

El impulso a los sectores dinámico exportadores ha ocasionado que regiones altamente especializadas en sectores no exportadores, se dispersen cada vez más de aquellas con una tradición exportadora, ampliando la brecha de crecimiento y acentuando las problemáticas sociales. De esta manera, las entidades más pobres se han caracterizado por poseer regiones con sectores económicos poco diversificados, y de poco interés para la política de gobierno, por no ser de exportación, como es el caso de la región económica número VII, del estado de Hidalgo.

Importancia del estudio regional: la región número VII del estado de Hidalgo.

En los últimos años los estudios que buscan explicar el desarrollo de las regiones han despertado el interés de los investigadores de las ciencias sociales. Hasta hace algunas décadas el concepto de región era poco utilizado como marco de referencia de estudio que dieran pauta al proceso de aplicación de medidas para la solución de problemas como el crecimiento y desarrollo de pequeñas fracciones de un territorio.
Boiser (1999), señala que una región es un territorio claramente organizado que contiene en términos reales los factores que impulsan su propio desarrollo independientemente de la escala [12]. Una región posee características sociales, económicas, culturales y políticas que son propias de sus pobladores y que propician identidad y cohesión social entre los lugareños que las conforman. El desarrollo de territorios bien definidos y establecidos como región, debe promoverse desde un análisis micro, logrando que sea la población que conforma las regiones su principal fuente de desarrollo, explotando no solo sus recursos naturales y productivos, sino, además su capacidad de organización. La teoría del desarrollo regional plantea a los agentes económicos como entes fundamentales del desarrollo.

Toda política regional se enfoca en tres objetivos básicos que son:

1) Desarrollar las áreas atrasadas,

2) Reducir los desequilibrios $y$,

3) Apoyar a las regiones con problemas sectoriales [6].

El impulso de los tres puntos anteriormente planteados, requieren la participación de un Estado promotor del desarrollo, que garantice no solo los derechos de propiedad, sino también, el derecho a un empleo decente, a la seguridad social, el respeto al medio ambiente y los derechos humanos. Estos elementos son contrarios a la idea neoliberal implementada en México desde los años ochenta, en donde el mercado debe ser el único promotor del crecimiento.

En estos 38 años de la implementación del neoliberalismo en México, la región número VII del estado de Hidalgo, conforma por los municipios de: Actopan, El Arenal, Francisco y Madero, Mixquiahuala, Progreso de Obregón, San Salvador y Santiago de Anaya; al igual que gran parte del territorio nacional ha vivido de manera directa los estragos de la política neoliberal. De acuerdo con la Encuesta Intercensal del INEGI 2015, esta región concentra al $8.1 \%$ de la población total del estado (233,362 personas), además, de poseer más de la mitad de su población en pobreza y pobreza extrema $(47.84 \%$ $4.9 \%$ respectivamente) [4].

Ante un gobierno incapaz de generar las alternativas de empleo que demanda la región, la migración internacional se ha convertido en la principal alternativa de la gente para lograr una vida digna, de esta forma el índice de intensidad migratoria México-Estados Unidos muestra el 29\% de los municipios se clasifican con un grado de alta intensidad migratoria, $57 \%$ con un nivel de intensidad migratoria medio, mientras que solo el $14 \%$ con un grado de intensidad migratoria bajo [13]. Las características que se viven en la región número VII del estado de Hidalgo la hacen una zona propicia para el desarrollo de esta investigación que busca mostrar que, en el contexto del 
neoliberalismo, en la región VII del estado de Hidalgo, se han favorecido sectores dinámico exportadores, sobre los sectores locales, lo que ha incrementado la pobreza y marginación que viven los habitantes de la región número VII del estado de Hidalgo.

\section{Análisis Shift-Share, generalidades}

Propuesta por Dunn en 1960, la metodología Shift - Share es una herramienta de análisis regional que tiene como objetivo la posibilidad de poder cuantificar los cambios que tienen las diferentes actividades económicas sobre el crecimiento de la misma actividad dentro de una región con relación al crecimiento nacional de la misma [5].

La ecuación clásica del modelo Shift-Share viene dada por:

$$
X^{\circ}{ }_{i j}-X_{i j}=\Delta X_{i j}=X_{i j} r+X_{i j}\left(r_{i}-r\right)+X_{i j}\left(r_{i j}-r_{i}\right)
$$

Donde:

$X_{i j}=$ magnitud económica de la región $\mathrm{j}$ en el sector i en el momento $\mathrm{t}$

$X^{\circ}{ }_{i j}=$ magnitud económica de la región j en el sector i en el momento $t+1$

$r=$ tasa de crecimiento de la magnitud económica a nivel nacional

$r_{i}=$ tasa de crecimiento de la magnitud económica para el sector i a nivel nacional

$r_{i j}=$ tasa de crecimiento de la magnitud económica para el sector i por la región j

La ecuación clásica Shift-Share divide el crecimiento en dos instantes del tiempo para una magnitud económica en tres componentes que son:

> Efecto nacional que permite distinguir los niveles de crecimiento de una rama de actividad específica y determinar si ésta crece a un ritmo mayor o menor que el nacional.

> El efecto sectorial indica si existe concentración de la producción en alguna industria de rápido crecimiento.

> Efecto competitivo que muestra si una región presenta mayor competitividad en relación al resto de las regiones en el crecimiento y evolución de los niveles de empleo.

Desde su aparición en 1960 la técnica Shift-Share ha sido revisada y modificada por diferentes autores como Esteban Marquillas (1972) y Nazara y Hewings (2004) con el propósito de eliminar algunos inconvenientes que presenta la primera formulación como la interdependencia de los efectos sectorial/regional, entre otros [14].

Una solución para el problema de interdependencia, fue la propuesta por Esteban Marquillas en 1972 al introducir el concepto de cambio homotético, definido como el valor que tendría la magnitud del sector i, en la región j si la estructura sectorial de la región coincidiera con la nacional [15]. De tal forma que expresado en términos del coeficiente de especialización se tiene:

$$
X_{i j}^{*}=\frac{X_{i j}}{C L_{i j}}
$$

Al incorporarlo a la identidad Shift-Share se obtiene la siguiente ecuación:

$\Delta X_{i j}=X_{i j} r+X_{i j}\left(r_{i}-r\right)+X_{i j}^{*}\left(r_{i j}-r_{i}\right)+\left(X_{i j}-X_{i j}^{*}\right)\left(r_{i j}-r_{i}\right)$

En la ecuación (3) se muestra el efecto competitivo neto, término de la derecha de la ecuación, que mide la ventaja o desventaja de cada sector en la región respecto al global. La técnica permite identificar los sectores económicos con mayor dinamismo en la región. Lo cual es concordante con el objetivo de esta investigación.

Para el desarrollo de la técnica se utilizó información proveniente de los Censos Económicos 2009 y 2014 publicados por el INEGI, correspondiente a los principales sectores económicos de los siete municipios que conforman la región, de esta manera el insumo fundamental fue el valor bruto de la producción por cada uno de los 15 sectores económicos que tienen presencia en la región.

\section{Resultados}

Comparando los resultados obtenidos a través del índice de localización, insumo indispensable para el desarrollo de la técnica Shift-Share, se encuentra que, de manera general, el número de sectores con una especialización mayor al promedio de la entidad hidalguense ha disminuido pasando de 6 sectores promedio en el año 2009 a 2 sectores promedio para el 20014, ver cuadro (1).

Cuadro (1)

Coeficiente de especialización (LQi), Región VII del estado de Hidalgo 2009 -2014 


\begin{tabular}{|c|c|c|c|c|}
\hline Municipio & Año & Sector de especialización & Lqi & \begin{tabular}{|c|} 
Total de \\
sectores \\
con LQi>1
\end{tabular} \\
\hline \multirow{2}{*}{ Actopan } & 2009 & $\begin{array}{l}\text { Servicios financieros y de } \\
\text { seguros }\end{array}$ & 2.14 & 9 \\
\hline & 2014 & $\begin{array}{l}\text { Servicios financieros y de } \\
\text { seguros }\end{array}$ & 4.03 & 9 \\
\hline \multirow[t]{2}{*}{ El Arenal } & 2009 & $\begin{array}{l}\text { Servicios de alojamiento } \\
\text { temporal y de preparación } \\
\text { de alimentos y bebidas }\end{array}$ & 6.28 & 2 \\
\hline & 2014 & & - & - \\
\hline \multirow[t]{2}{*}{$\begin{array}{c}\text { Francisco I. } \\
\text { Madero }\end{array}$} & 2009 & $\begin{array}{l}\text { Servicios de alojamiento } \\
\text { temporal y de preparación } \\
\text { de alimentos y bebidas }\end{array}$ & 3.90 & 7 \\
\hline & 2014 & Minería & 1.28 & 1 \\
\hline \multirow{2}{*}{$\begin{array}{l}\text { Mixquiahuala de } \\
\text { Juárez }\end{array}$} & 2009 & Comercio al por mayor & 4.43 & 6 \\
\hline & 2014 & Comercio al por mayor & 2.67 & 2 \\
\hline \multirow{2}{*}{$\begin{array}{l}\text { Progreso de } \\
\text { Obregón }\end{array}$} & 2009 & $\begin{array}{l}\text { Servicios financieros y de } \\
\text { seguros }\end{array}$ & 2.43 & 7 \\
\hline & 2014 & Minería & 30.63 & 2 \\
\hline \multirow[t]{2}{*}{ San Salvador } & 2009 & $\begin{array}{l}\text { Otros servicios excepto } \\
\text { actividades } \\
\text { gubernamentales }\end{array}$ & 2.75 & 6 \\
\hline & 2014 & - & - & - \\
\hline \multirow[b]{2}{*}{$\begin{array}{l}\text { Santiago de } \\
\text { Anaya }\end{array}$} & 2009 & $\begin{array}{l}\text { Servicios inmobiliarios y de } \\
\text { alquiler de bienes muebles e } \\
\text { intangibles }\end{array}$ & 9.69 & 4 \\
\hline & 2014 & $\begin{array}{l}\text { Servicios de apoyo a los } \\
\text { negocios y manejo de } \\
\text { desechos y servicios de } \\
\text { remediación }\end{array}$ & 4.70 & 1 \\
\hline
\end{tabular}

Fuente: Elaboración propia con base en Censos Económicos 2009, 2014. INEGI.

Así mismo, puede observarse que la mayoría de los municipios se encuentran especializados en el sector terciario y, solo los municipios de Francisco I. Madero y Progreso de Obregón tienen una especialización en la minería. El coeficiente de especialización nos muestra el abandono de actividades de mayor empleabilidad en la región número VII, como lo es el campo y el comercio al por menor. Lo que demuestra una mala estrategia de la política pública para impulsar el desarrollo de la región.

Además, al calcular el efecto Total, se encuentra que este es negativo para todos los municipios, lo que significa que ninguno de ellos ha crecido más que la media estatal (Ver cuadro 2).

\section{Cuadro (2)}

Efecto Total, región VII del estado de Hidalgo:2009-2014

\begin{tabular}{cl}
\hline Municipio & \multicolumn{1}{c}{ Efecto Total } \\
\hline Actopan & -617.94 \\
El Arenal & -120.65 \\
Francisco I madero & -12.50 \\
Mixquiahuala de Juárez & -531.90 \\
Progreso de obregón & -83.50 \\
San Salvador & -2.31 \\
Santiago de Anaya & -6327.11 \\
\hline
\end{tabular}

Fuente: Elaboración propia con base en Censos Económicos 2009, 2014. INEGI.

Con referencia al efecto diferencial obtenido en los municipios de la región número VII del estado de Hidalgo, cuadro (3), se observa que solamente dos sectores económicos tuvieron un crecimiento mayor acorde con el dinamismo sectorial. Los sectores económicos más dinámicos fueron:

1) La industria manufacturera $y$,

2) El sector servicios de apoyo a los negocios y manejo de desechos y servicios de remediación.

La industria manufacturera, resulto ser uno de los sectores económicos más dinámicos, de hecho, fue el único que tuvo una tendencia positiva en todos los municipios que conforman la región. Este resultado es congruente con la política exportadora desarrollado durante la era neoliberal, por lo que se ha convertido en el eje del desarrollo del país y sus regiones, no importando aquellos sectores que contribuyen a mejorar el mercado interno y fomentan el nivel de empleo.

De la misma manera, el sector servicios de apoyo a los negocios y manejo de desechos y servicios de remediación, tiene una fuerte presencia en los municipios de la región, a excepción del municipio de Progreso de Obregón, donde su participación no resulto ser significativa. Es importante destacar que en este sector de actividad se encuentra el subsector de apoyo a negocios, subsector que contribuye con el total de actividades productivas para este sector económico. Al igual que el sector manufacturero, esta última actividad representa un elemento clave para el desarrollo de la política neoliberal.

Cuadro (3)

Región VII de Hidalgo, 2009-2014, Componente diferencial.

\begin{tabular}{|l|r|r|}
\hline \multicolumn{3}{|c|}{ Componente diferencial } \\
\hline & \multicolumn{1}{|c|}{$\begin{array}{l}\text { Servicios de } \\
\text { apoyo a los } \\
\text { negocios y } \\
\text { manejo de } \\
\text { desechos y } \\
\text { servicios de } \\
\text { remediación }\end{array}$} \\
Municipio & $\begin{array}{l}\text { Industrias } \\
\text { manufactureras }\end{array}$ & 20.29 \\
\hline Actopan & 26.09 & 0.27 \\
\hline El Arenal & 2.89 & 1.18 \\
\hline $\begin{array}{l}\text { Francisco I. } \\
\text { Madero }\end{array}$ & 13.20 & 2.09 \\
\hline $\begin{array}{l}\text { Mixquiahuala } \\
\text { de Juárez }\end{array}$ & 152.60 & 0.84 \\
\hline $\begin{array}{l}\text { Progreso de } \\
\text { Obregón }\end{array}$ & 48.83 & - \\
\hline San Salvador & 5.93 & 0.55 \\
\hline $\begin{array}{l}\text { Santiago de } \\
\text { Anaya }\end{array}$ & 5.13 & 82.64 \\
\hline
\end{tabular}

Fuente: Elaboración propia con base en Censos Económicos 2009, 2014. INEGI. 
En el cuadro (4), se presentan los efectos diferencial y estructural total, obtenidos a través de la técnica Shift and Share, los resultados nos indican que los municipios que conforman la región número VII del estado de Hidalgo, se han clasificado en regiones perdedoras, es decir, su crecimiento es menor que el promedio de la entidad. Este resultado se obtiene a partir de la tipología de regiones con análisis estructural, que clasifica las regiones en 6 tipos, 3 con efecto total positivo y tres con efecto total negativo [16].

\section{Cuadro (4)}

Región VII de Hidalgo, 2009-2014, tipología de regiones con análisis diferencial estructural.

\begin{tabular}{|c|c|c|c|}
\hline Efecto Total Negativo & $\begin{array}{c}\text { Efecto } \\
\text { Diferencial } \\
\text { Total } \\
\end{array}$ & $\begin{array}{c}\text { Efecto } \\
\text { estructural }\end{array}$ & Región tipo \\
\hline Actopan & 119.37 & 737.31 & III B \\
\hline El Arenal & 38.39 & 82.26 & IV \\
\hline Francisco I. Madero & 69.82 & 82.31 & III B \\
\hline Mixquiahuala de Juárez & 25.81 & 506.09 & IV \\
\hline Progreso de Obregón & 282.65 & 366.15 & III B \\
\hline San Salvador & 20.84 & 23.15 & III B \\
\hline Santiago de Anaya & 91.01 & - $\quad 6,418.12$ & III B \\
\hline
\end{tabular}

Fuente: Elaboración propia con base en Censos Económicos 2009, 2014. INEGI.

Resulta eminente que las políticas neoliberales, han impactado de manera negativa a la región número VII del estado de Hidalgo lo que ha ocasionado el apoyo a sectores económicos, considerados estratégicos para impulsar la dinámica exportadora del país sin favorecer, el desarrollo regional, si nomás bien abandonando sectores estratégicos para el desarrollo local como el agrícola y el comercio al por menor.

\section{Conclusiones}

Bajo el contexto del modelo neoliberal implementado en nuestro país en la década de los 80 's, se han favorecido sectores que tienen como objetivo los grandes mercados internacionales, sin importar el impacto que se tiene sobre el crecimiento de la región, la cual en el caso del presente estudio fue negativo. La manufactura como sector dinámico regional tiene diferencias notables en su existir, por ejemplo, se favorece la industria maquiladora de exportación, sin importar los pocos encadenamientos productivos que puedan generar en el país. Aunado a lo anterior la política de bajos salarios y precarias condiciones laborales, permitidas por la legislación vigente vuelven cada vez más vulnerables a los trabajadores nacionales.

La región número VII del estado de Hidalgo, requiere diversificar sus actividades productivas, pues como se pudo observar en el análisis realizado solamente los municipios de Actopan, conservo los siete sectores en los cuales se encontraba especializado en el año 2009 , todos los demás municipios redujeron su especialización lo que hizo que su crecimiento económico se concentrara solamente en una o dos actividades económicas, generando una dependencia casi total, al buen funcionamiento de las actividades sobresalientes.

Ante este contexto, se requiere generar políticas públicas que orienten el crecimiento económico a diversos sectores de actividad económica. La diversificación de la producción en diversas actividades nos hace ser menos dependientes a los mercados externos y por tanto menos vulnerables a los vaivenes internacionales. Se debe incentivar actividades que se caracterizan por ser intensivas en mano de obra y de poco valor agregado, como, el campo, la minería, la construcción, el comercio al por menor, su tecnificación es imprescindible para lograr que miles de mexicanos que se dedican a estas actividades productivas, las vislumbren como una de sus alternativas de desarrollo.

Es imprescindible que veamos en la colectividad una forma de impulsar el desarrollo, hoy debemos alejarnos de esas ideas que plantean el individualismo como la única manera de lograr el bienestar de los seres humanos, requerimos participan en la construcción del país que queremos, un México con oportunidades de empleo, sin violencia y respetuoso de los derechos humanos y del medio ambiente.

\section{Referencias}

[1] Palley, Thomas I. (2005). Del keynesianismo al neoliberalismo: paradigmas cambiantes en economía. Economía UNAM, 2(4), 138-148.

[2] Méndez, M. J. S. 1998. El neoliberalismo en México iéxito o fracaso? Revista de Contaduría y Administración. Universidad Nacional Autónoma de México (UNAM). 191:56-65.

[3] Salas-Luévano, M (2009) Migración y feminización de la población rural 2000-2005. El caso de atitanac y la encarnación, villanueva, zac. Mexico, Universidad Autónoma de Zacatecas.

[4] CONEVAL, 2015. Pobreza a nivel Municipal 2015. Visitado por última vez el 14 de Diciembre del 2019. Disponible en https://www.coneval.org.mx/coordinacion/entidades/Hidalgo/Paginas/ pobreza_municipal2015.aspx..

[5] Bolaños, R. L (2010). "Metodología Shift-Share y su posible aplicación al sistema bancario". Universidad de Costa Rica: Instituto de Investigación en Ciencias Económicas. Proyecto: 721-A9-163

[6] Merchand-Rojas, M.A. (2007). Teorías y conceptos de Economía Regional y estudios de caso. Guadalajara: Universidad de Guadalajara.

[7] Gobierno del Estado de Hidalgo, (2011). Decreto que determina la regionalización de los municipios del Estado libre y soberano de Hidalgo. Visitado por última vez el 17 de agosto de 2020. Disponible 
en:

http://intranet.ehidalgo.gob.mx/NormatecaE/Archivos/archivo1886.pdf

[8] Harvey, D. (2007). Breve historia del neoliberalismo (No. 49). Ediciones Akal.

[9] Sunkel, O., \& Paz, P. (1999). El subdesarrollo latinoamericano y la teoría del desarrollo. 1st edición. Méxixo: Siglo XXI.

[10] Argandoña, A. (1990). El pensamiento económico de Milton Friedman. Universidad de Navarra.

[11] Hayek, F. (1995). Camino de servidumbre, Madrid, Alianza

[12] Boisier, S. Desarrollo (local): ¿ de qué estamos hablando. Santiago de Chile, 1999, vol. 28

[13] CONAPO (2010). Anexo B. Índices de intensidad migratoria México-Estados Unidos por entidad federativa y municipio. Visitado por última vez el 14 de Diciembre de 2019. Disponible en: http://www.conapo.gob.mx/work/models/CONAPO/intensidad_migrat oria/anexos/Anexo_B1.pdf

[14] Márquez, M. A., Ramajo, J., \& Hewings, G. J. (2009) Incorporating sectoral structure into Shift-Share analysis. Growth and change, 40(4), 594-618.

[15] Mayor, M., \& López, A. J. (2005). El análisis Shift-Share espacial: nuevos desarrollos. VIII Encuentro de economía aplicada-España, Facultad de Economía y Empresas, Universidad de Murcia, España.

[16] Gaona , E., Vázquez, R. \& Rodríguez, E. (2019). Hidalgo: Desafíos para el desarrollo. México, Editorial UAEH 